\title{
Examining the Validity of the Lexical Access Time Test (LEXATT2)
}

\author{
Tatsuo Iso \\ Reitaku University \\ doi: http://dx.doi.org/10.7820/vli.v01.1.iso
}

\begin{abstract}
This study aimed to investigate the validity of the Lexical Access Time Test (LEXATT2). The first step was to examine the test results to determine if it was able to differentiate between participants with different English proficiency levels. The results were further analyzed to see if longer target words elicited slower lexical access times. The results of the 119 participants indicated promise in that LEXATT2 established an ability to distinguish proficiency levels to some extent. Further, it was found that LEXATT2 elicited slower lexical access time from the participants with lower English proficiency.
\end{abstract}

Keywords: lexical access; fluent reading; automaticity of lexical access; word recognition; meaning retrieval; lexical access time test; computerized language testing.

\section{Introduction}

In recent years, greater attention has been paid to automaticity of lexical access. Though the research on vocabulary knowledge has focused mainly on vocabulary size, having automatic access to words and their meanings is almost as important in performance. The importance has been recognized in reading research where automatic lexical access is one of the most fundamental processes for fluent reading (Grabe, 2009; Grabe \& Stoller, 2002; Koda, 2005). Along with vocabulary size and organization, automaticity of lexical access is now considered a dimension of vocabulary knowledge (Meara, 2005).

Despite its importance, how we measure the automaticity of lexical access is at issue. To address this problem, this study aimed to introduce my latest attempt at building a test of the automaticity of lexical access as well as to validate the Lexical Access Time Test (LEXATT2).

\section{Description of the LEXATT2}

\subsection{What it Measures}

Lexical Access Time Test is a computer program which aims to measure the time that it takes to recognize a word and retrieve its meaning. As illustrated in Figure 1, lexical access time is defined as the difference between the reaction time for the verbal stimulus (RT (VL)) and the mean reaction time for nonverbal stimulus (MRT (NV)). Admitting that speed is merely one aspect of automaticity (Segalowitz \& Segalowitz, 1993), LEXATT2 measures the speed of lexical access to keep the test as simple as possible. 


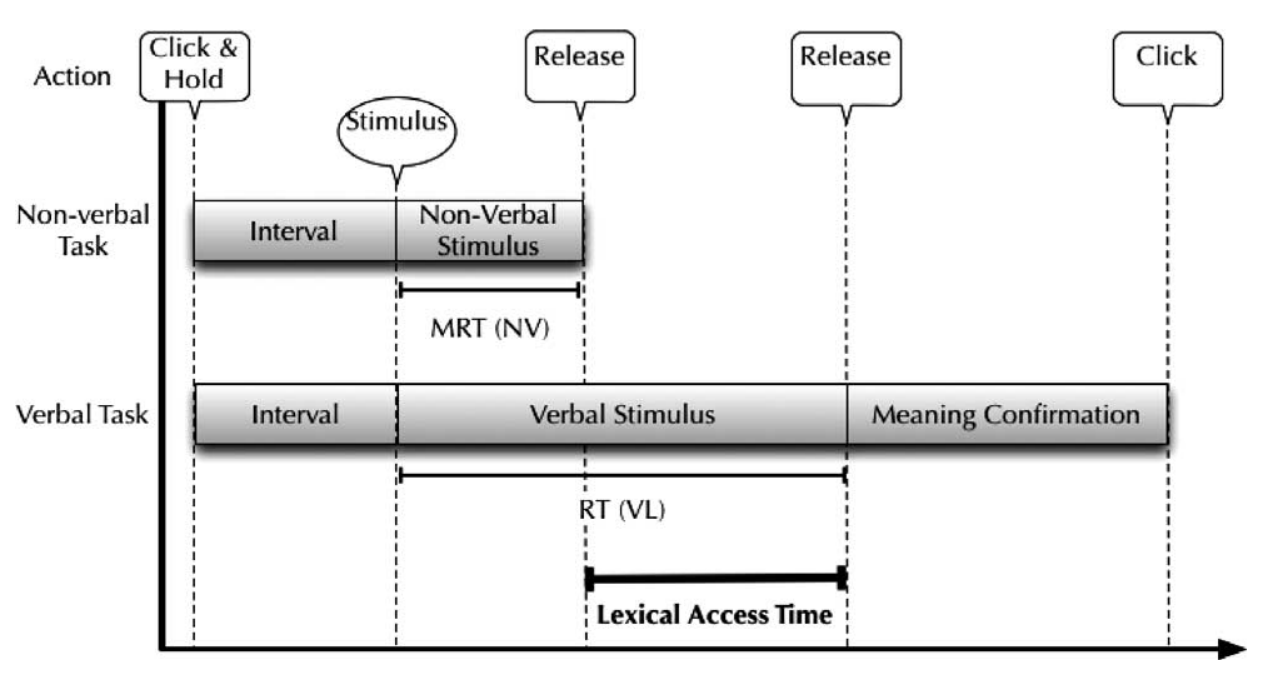

Figure 1. Lexical access time in LEXATT2.

\subsection{How it Measures Lexical Access Time}

There are two tasks in LEXATT2. The first is a nonverbal reaction time task. Five instances of nonverbal reaction time are measured and averaged to produce MRT (NV).

The second task consists of two stages: verbal reaction time stage and meaning confirmation stage. The verbal reaction time stage is the same as the nonverbal reaction time task except the stimuli are English words (see below for the target words). In the meaning confirmation stage that immediately follows the verbal reaction time stage, the test takers are asked to choose the meaning or synonym of the target word displayed in the previous stage. This stage is necessary to make sure the test taker retrieve a word meaning when reacting to the verbal stimulus, so that LEXATT2 is not measuring word recognition time.

\subsection{Target Words}

There are 45 target words in LEXATT2. They are high-frequency words, within the 2000 level of the JACET list of 8000 basic words (JACET, 2003). The target words are also limited to 4, 6, or 8 letters in length, and there are 15 words each. The actual target words are as follows: band, bite, boot, cave, cash, debt, sail, hang, seed, lord, mail, meat, pack, pole, tale, afford, actual, attend, export, cancer, charge, divide, fairly, formal, insist, lovely, bottle, manner, threat, creature, marriage, customer, identity, original, security, response, complain, exciting, positive, strength, maintain, division, presence, laughter, persuade.

\section{The Study}

\subsection{Purpose}

As previously mentioned, this study aims to investigate the validity of LEXATT2. For this purpose, the following research questions were asked.

1. Does LEXATT2 distinguish test takers of different English proficiency levels?

2. Does LEXATT2 elicit slower lexical access time for longer words? If so, how does the length of words interact with the test takers' proficiency levels? 
The assumption of the first question was that if the automaticity of lexical access was part of vocabulary knowledge, it would improve along with the proficiency. Therefore, LEXATT2 was expected to elicit shorter lexical access times from higher-proficiency groups. As for the second question, it was assumed that LEXATT2 would elicit longer lexical access times for longer words if the test was sufficiently accurate.

\subsection{Participants}

There were a total of 194 participants in the study. The participants were divided into four groups based on their proficiency levels: native speakers (NS), Japanese Teachers of English (JTE), higher-level university students (Stu-H), and lower-level university students (Stu-L). The cutoff point of the higher-proficiency students was 470 on Test of English for International Communication (TOEIC). Some of these subjects were eliminated after taking LEXATT2 due to the low percentages of correct answers (below 70\% during the meaning confirmation stage).

Table 1 shows the distribution of the remaining 119 participants. As for the proficiency levels of the NS and the JTE groups, it was assumed that the NS group was the highest in proficiency followed by JTE, who were further assumed to have higher proficiency than the Stu-H. The two student groups were significantly different with regard to their proficiency levels based on TOEIC scores.

Table 1. Distribution of the Participants

\begin{tabular}{llll}
\hline & $N$ & Mean TOEIC & Notes \\
\hline NS & 27 & N/A & \\
JTE & 22 & N/A & \\
Stu-H & 29 & 561.21 & $t(68)=11.34, p<0.01$ \\
Stu-L & 41 & 387.57 & \\
\hline
\end{tabular}

\section{Results}

Prior to test validation, the reliability of the test was calculated with correct/ incorrect responses in the meaning confirmation stage. The Cronbach's alpha was 0.80 , and the test was considered reliable enough.

To answer the first research question, the four groups were compared in terms of the mean lexical access time (see Table 2). As illustrated in Figure 2, the lexical access time seemed to be shorter for the higher-proficiency group. A one-way ANOVA revealed that proficiency had a significant effect on the lexical access

Table 2. Group Means for Overall Lexical Access Time and for Four-, Six-, and Eight-Letter Words

\begin{tabular}{lllllll}
\hline & N & $\begin{array}{l}\text { Lexical access } \\
\text { time }(\mathrm{ms})\end{array}$ & SD & $\begin{array}{l}\text { Four letters } \\
(\mathrm{ms})\end{array}$ & $\begin{array}{l}\text { Six letters } \\
(\mathrm{ms})\end{array}$ & $\begin{array}{l}\text { Eight letters } \\
(\mathrm{ms})\end{array}$ \\
\hline NS & 27 & 287.37 & 184.43 & 282.47 & 283.85 & 295.41 \\
JTE & 22 & 357.00 & 199.65 & 349.41 & 342.79 & 376.80 \\
Stu-H & 29 & 446.00 & 299.50 & 330.86 & 409.78 & 534.50 \\
Stu-L & 41 & 537.15 & 341.31 & 406.23 & 570.10 & 798.05 \\
\hline
\end{tabular}


time with $F(3,115)=4.92, p<0.001, \eta^{2}=0.11$, and an LSD (least significant difference) post hoc test revealed that the differences between the NS and the Stu-H, the NS and the Stu-L, and the JTE and the Stu-L were all significant.

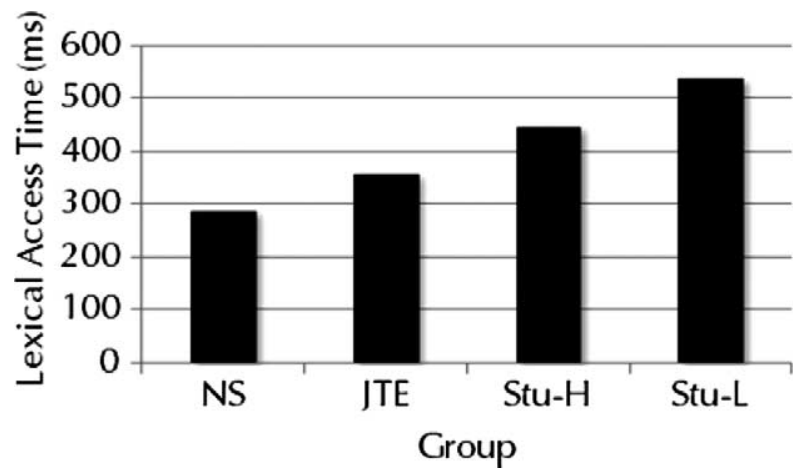

Figure 2. Lexical access time for each group.

As for the second research question, which asked if the length of the target words affected the lexical access time, the results are also shown in Table 2 and Figure 3. A two-way ANOVA with mixed design revealed the interaction of the proficiency group and the word length was significant $(F(6,230)=15.58$, $p<0.01, \eta_{p}^{2}=0.29$ ). An LSD post hoc test showed that the lexical access time of the six-letter words by the Stu-L group was significantly slower than those of the other groups. This difference intensified with the eight-letter words.

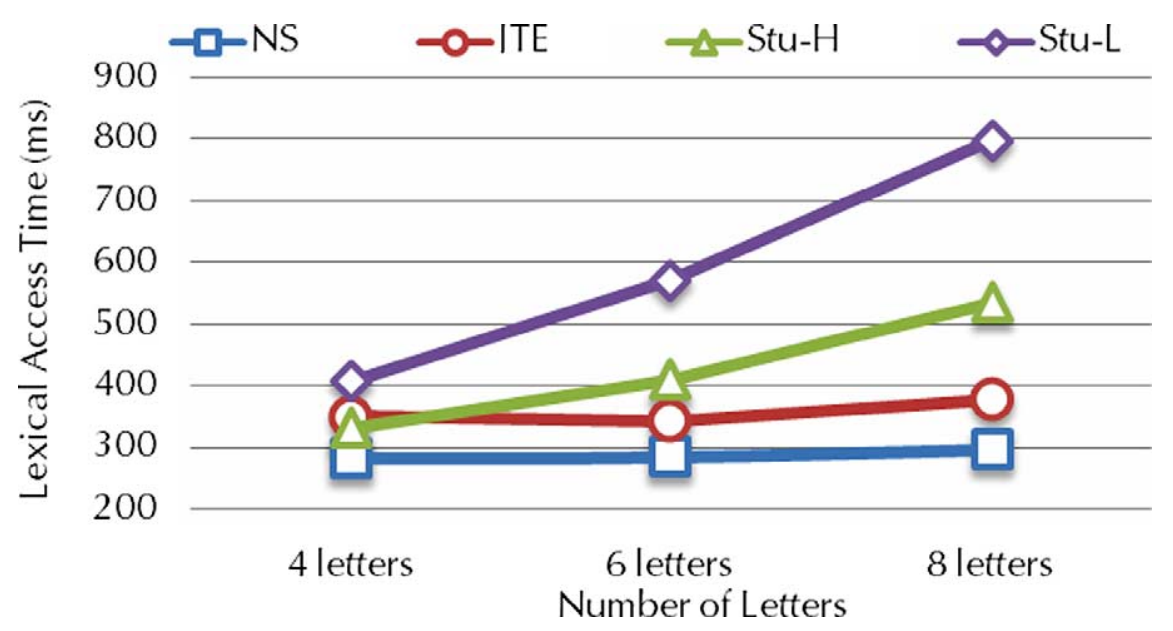

Figure 3. Lexical access time of three types of target words.

Another tendency was discovered when the word-length effect was compared within the groups. While the NS's and the JTE's performance was unaffected by the length of the target words, the Stu-H and the Stu-L demonstrated significantly slower lexical access time for longer words, with the exception of the difference between the Stu-H group's four- and six-letter words.

\section{Discussion}

The validity of LEXATT2 is discussed in light of the results shown above. With regard to the comparison of the overall lexical access time among proficiency 
groups, LEXATT2 showed an ability to distinguish the highest proficiency group from lower ones. However, it was not successful in distinguishing two higher proficient groups, namely the NS and the JTE. Even so, this does not necessarily completely negate the validity of LEXATT2, as the difference in proficiency between these groups was obscure to start with. Further examinations with larger number of participants with wider ranges of tangible, not assumed, proficiency levels are needed.

In terms of the analysis of the effect of the word length, the results of the lower-level participants were in accordance with intuitive expectations. LEXATT2 elicited slower lexical access time for longer words. Such an effect, however, disappeared for the higher-level participants. One may argue that this threatens the validity of the test. Yet, it is highly possible that high enough proficiency mitigates the effect of word length in lexical access. Yet another possibility is that the highproficiency participants in this study knew the target words so well that the word length did not affect the lexical access time. Including even longer words may bring about clearer pictures.

Although LEXATT2 was found to have high potential, the findings also suggest the need for improvements. As mentioned above, including longer words as stimuli may be necessary. Further, there is a need for an infallible system to prevent test takers from placing too much emphasis on speed in lieu of accuracy during lexical access. About $40 \%$ of the original participants (mostly university students) did not choose correct meanings of target words due to the fact that they did not pay attention to the meaning of the target word in the verbal reaction time stage. Admitting that there is room for aforementioned improvement, the results of this study seem to demonstrate the promise of LEXATT2 as a tool for measuring English learners' lexical access speed.

\section{References}

Grabe, W. (2009). Reading in a second language: Moving from theory to practice. New York, NY: Cambridge University Press.

Grabe, W., \& Stoller, F.L. (2002). Teaching and researching reading. London: Pearson Education.

JACET Basic Word Revision Committee (2003). JACET list of 8000 basic words. Tokyo: Japan Association of College English Teachers.

Koda, K. (2005). Insights into second language reading. Cambridge: Cambridge University Press.

Meara, P. (2005). Designing vocabulary tests for English, Spanish, and other languages. In C.S. Butler, M.A. Gomez-Gonzalez \& S. Doval-Suarez (Eds.), The dynamics of language use: Functional and contrastive perspectives (pp. 271-285). Amsterdam: John Benjamins.

Segalowitz, N., \& Segalowitz, S.J. (1993). Skilled performance, practice, and the differentiation of speed-up from automatization effects: Evidence from second language word recognition. Applied Psycholinguistics, 14, 369-385. doi:10.1017/S0142716400010845. 